\title{
MANAGING S/W DESIGN CHANGES USING C.R. DESIGNER
}

\author{
Siddharth Dixit ${ }^{1}$, Ranu Ratnawat ${ }^{2}$, Umesh Sharma ${ }^{3}$, \\ Praveen Kr. Srivastava ${ }^{4}$ \\ Software Design Group, \\ Center for Development of Advanced Computing(C-DAC), Noida, India \\ ${ }^{1}$ siddharthdixit@cdac.in, ${ }^{2}$ ranuratnawat@cdac.in \\ ${ }^{3}$ umeshsharma@cdac.in, 4pksrivastava@cdac.in
}

\begin{abstract}
The development of any software product depends on how efficiently design documents are created. The various kind of design document which are required to be created for the development of software product are High level Design (HLD), Low Level Design (LLD) and Change Request Design (CRD) Document . Low level design document gives the design of the actual software application. Low level design document is based on High Level Design document [2]. After an application is implemented at client site, there can be changes which the client may ask during the implementation or maintenance phase. For such changes, Change Request Design documents are created. A good design document will make the application very easy to develop/maintain by the developer. CR Designer tool is designed and developed to create a standard Change Request Design (CRD) documents and tracing the changes done in processes of the module in different versions of CRD document released. This paper presents a new dimension tool for Change Request Design Document.
\end{abstract}

\section{KEYWORDS}

Change Request Design Document, Document Type, Document format standardization, software Design document, CR Designer

\section{INTRODUCTION}

Software Design phase is the specification of software structure at various level of details [3]. Without reliable design documentation, significant software systems become less accessible to software engineers because structural information is buried in the intricate implementation source code [3]. As per Agile Design best practices, designers should research iteration $n+2$, design iteration $\mathrm{n}+1$, support iteration $\mathrm{n}$ and review iteration $\mathrm{n}-1[4]$.

Changes are inherent in the development of most software systems. Change Request process is one of the main task in software configuration management. A change request is a request to add a new feature to the product or to enhance an existing specification due to a defect or failure, a change request is created to modify the existing requirement specification. A CRD document describes the detailed design description for handling the changed requirement in an existing system.

DOI : 10.5121/ijsea.2013.4207 
Because requirements are changeable throughout the software development lifecycle, and requirements specification is a dynamic process, requirements are not often complete until the end of the product implementation therefore the CRD document can be generated.

A change request is declarative, i.e. it states what needs to be accomplished, but leaves out how the change should be carried out. Important elements of a change request are an ID, the customer (ID), the deadline (if applicable), an indication whether the change is required or optional, the change type (often chosen from a domain-specific ontology) and a change abstract, which is a piece of narrative [12]

Change requests typically originate from one of five sources: (i) problem reports that identify bugs that must be fixed, which forms the most common source, (ii) system enhancement requests from users, (iii) events in the development of other systems, (iv) changes in underlying structure and/or standards (e.g. in software development this could be a new operating system), and (v) demands from senior management.

The simultaneous explosion of information, the integration of technology, and the continuous evolution from software-intensive systems to ultra-large-scale (ULS) systems require new and innovative approaches for building, running, and managing software systems [9].

This paper describes the functionality of Change Request Designer Tool developed for CRD document Creation. It is organized as follows Section 2 describes the problems in current CRD document creation style for preparing CRD documents. Section 3 discusses the features of the CRD Designer Tool. Section 4 contains the Implementation details of this tool and 5 conclude the paper.

\section{Problems in Current change REQuest Design Document CREATION}

Currently all the CRD document and associated user interfaces created manually on MS Word editor. CRD Document created manually has many disadvantages. Proper standardization is not followed as each designer follows their own language and style of writing. If one CR is released multiple times with some changes, versions of CR released cannot be maintained. Once a CRD document is created and if some change is required across all user interfaces then all the screens are required to be redesigned / modified.CR tracking at process level cannot be done. That is if we want to check how many times a process (P1) is modified, we cannot track this with the current system.

\section{Change Request Designer tool AND its features}

CR Designer Tool is a J2EE Web based utility, used for making Change Request Design documents. In Software Maintenance phase, what happens during the rest of the software's life: changes, correction, additions, moves to a different computing platform and more[11] is taken care of. CR Designer Tool helps in making design document which for such design level changes.

There are following features in the CR Designer tool:-

a. The CRD document can be easily created and modified using this tool.

b. View of CRD document remains same among user leading to standardize format of design document. 
c. Document version at the time of release is maintained.

d. Tracking of CRD details of Processes in module in different versions of Change Request Document.

e. Process and module related affected image upload facility

f. Change Request wise/ Requirement wise design document viewing facility.

g. User can upload Existing CRD document created on Word Editor.

\section{IMPLEMENTATION OF C R DESIGNER TOOL}

Interactive design is heavily focused on satisfying the needs and desires of the people who will use the product [5]. A change is provided from the client in form of a document which contains multiple modification/New requirements to be done in the modules of a project.

CR Designer Tool groups module wise multiple requirements in one CR design document. Each requirement is further subdivided into process level changes. Many times new processes are required to be made.

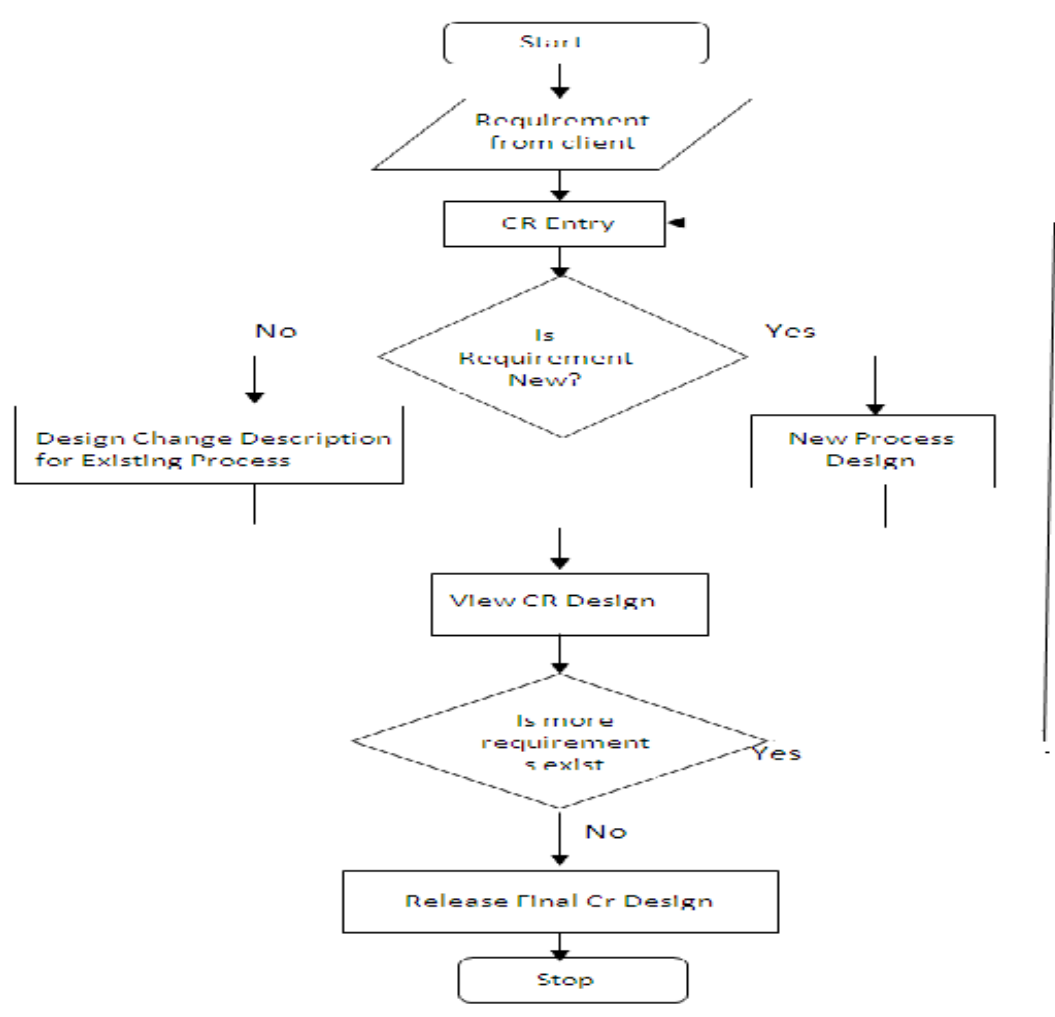

Figure 1: CR Designer Flow

These CR can be viewed, modified and released for development. Version of CR released is maintained and Tracking of all releases can be done.

This tool has following processes to fulfill the design requirements:-

- CR Detail Entry

- CR Design Creator

- View CR Design 
- Release Final Change Request Design View

- CR Upload

- Trace Change Request Design Document

\subsection{CR Detail Entry}

This process is used to create new CR document for a module. It records detail of change request and creation date of the change request.

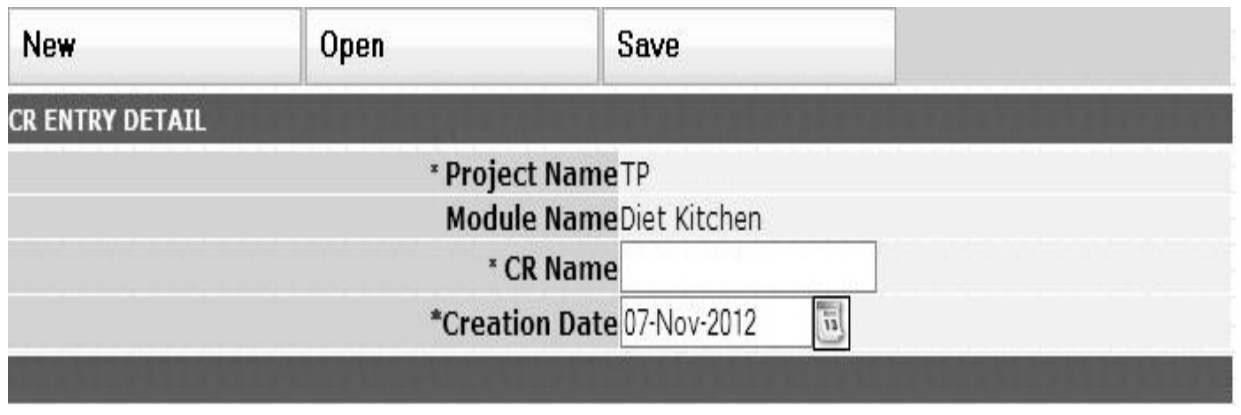

Figure 2: CR Detail Entry

\subsection{CR Design Creator}

This is a main process for CR Design document creation. In this process requirement wise design document is created and changes are specified at process level. Key feature of this process are:-

1) In case of change in existing process, provision to make the documentation for Process Change Description and database Schema Change Description parameters that are mandatory for a CR Design Document

2) In case if requirement demands for a new process, provision to make the documentation for Process Description, Entry Criteria, Exit Criteria, Table Used parameters for a CR Design Document

3) Provision to upload the Interface Affected, flow diagram, Use Case Diagram, Class Diagram etc.

4) Viewing the resultant requirement level documentation, that is all the process design documentation done for the requirement.

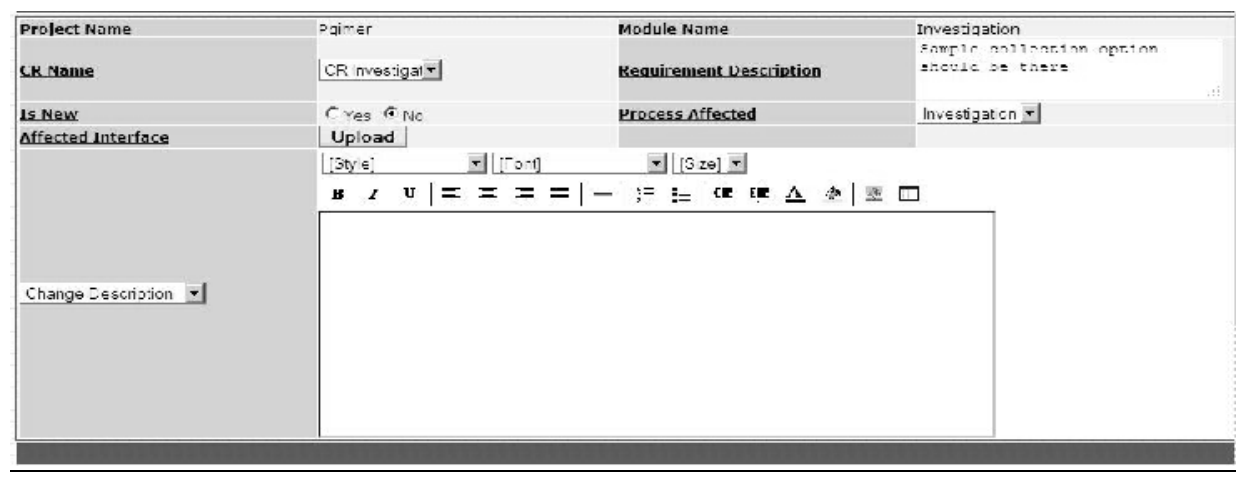

Figure 3: CR Design Creator 


\subsection{View CR Design}

This process is used to generate the final CR Design Document. The process is used to view requirement wise as well as complete $\mathrm{CR}$ wise design created for a module. While implementing the utility, it is found that certain changes in document are required. For handling such changes CR Design view is used for reviewing and editing the CRD document created by CR Design Creator process.

\begin{tabular}{|c|c|c|c|}
\hline \multicolumn{2}{|l|}{ NEW } & View Complete CRD & \\
\hline \multicolumn{4}{|c|}{ IIEW DESIGN DOCUMENT } \\
\hline \multicolumn{2}{|r|}{ Project NameTP } & \multicolumn{2}{|c|}{ Module NameDiet Kitchen } \\
\hline \multicolumn{4}{|c|}{ View Document $C$ LLD Document 6 CR Document } \\
\hline \multicolumn{4}{|c|}{ CR Name Diet Kitchen Issue $\nabla$} \\
\hline \multicolumn{4}{|c|}{ C.R.D. CONTENTS } \\
\hline \multicolumn{3}{|c|}{$\begin{array}{l}\text { Point no.-CR/GGSH/163, CR/GGSH/164, CR/GGSH/165, CR/GGSH/166 In Diet } \\
\text { Calculator process- } 1 . \text { By... }\end{array}$} & View Doc. \\
\hline
\end{tabular}

Figure 4: View CR Design

\subsection{Release Final Change Request Design View}

This process is the final output of the utility. This is used to view complete CRD document of the Module in HTML format. This document will be released to the development team for making changes in the module as per CRD.

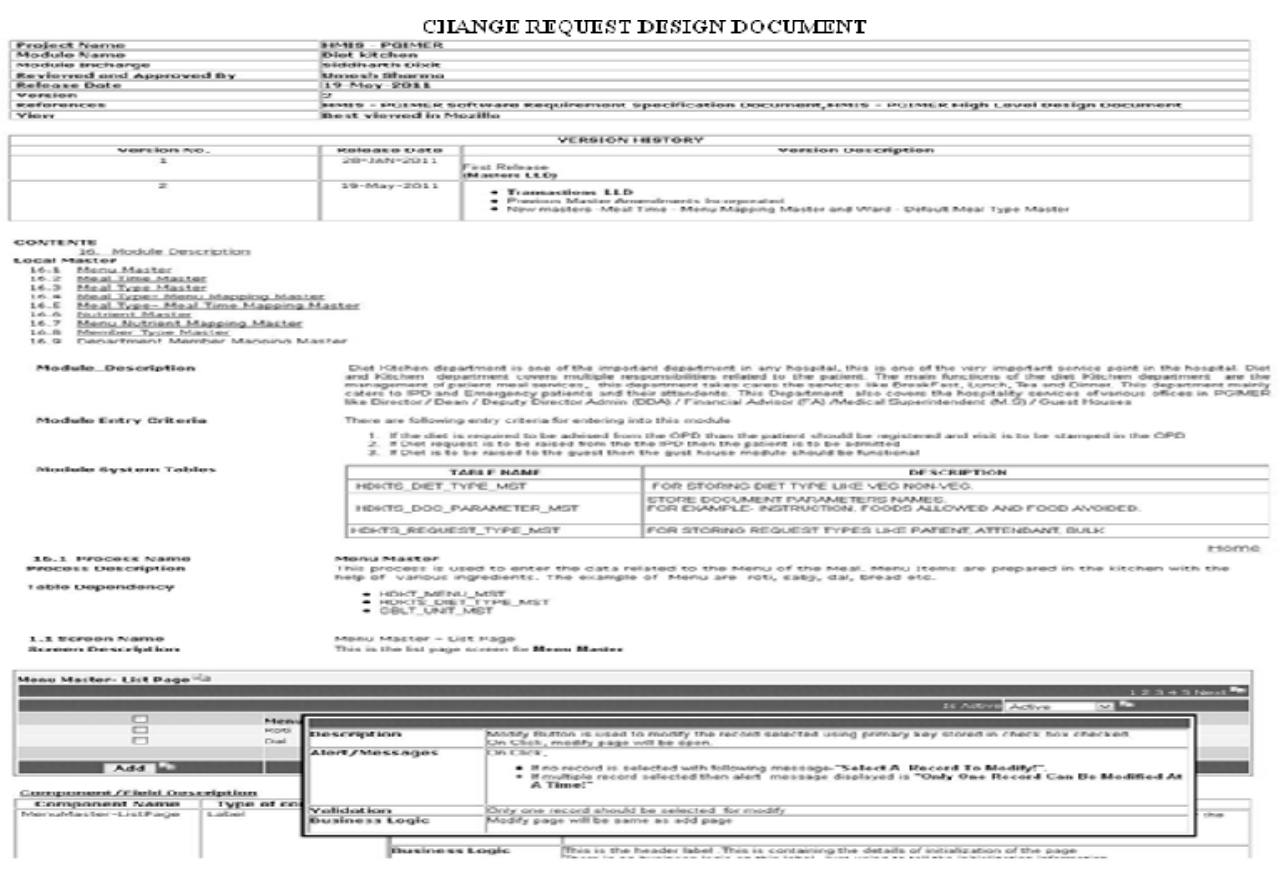

Figure 5: Release Final Change Request Design View 


\subsection{Trace Change Request Design Document}

Tracking the maintenance behaviour of the software product is very complex. This is precisely the reason that predicting the cost and risk associated with maintenance after delivery is extremely difficult which is widely acknowledged by the researchers and practitioners [8].

The goal of a traceability matrix is -

1. To make sure that the approved requirements are addressed/covered in all phases of development.

2. That each document should be traceable: Written test cases should be traceable to its requirement specification. If there is new version then updated test cases should be traceable with that [6].

A CRD document undergoes multiple iterations during its Design, development and maintenance. The tool therefore takes care of the versioning issues and tracking of changes done in processes of module. User can view all the versions of CRD Document released.

Tracking of changes done can be at both, module level as well as process level. At module level, this utility can keep track of how many change request design document are made, creation date, release date, created and approved by person's name.

User can also trace for changes done in selected process in different versions of CRD Document. For tracking process level changes, user can search for the process and the CRD documents that contain the process will be displayed.

\begin{tabular}{|c|c|c|c|c|}
\hline Module Name & Latest Version ND. & Release Date & Version Description & Support Document(s) \\
\hline contlgency & 1.1 & 06-Feb-2012 & (v) & \\
\hline Cash NFee & 1.1 & 02-Feb-2012 & (v) & \\
\hline Sanitation & 3.1 & 03-Jan-2012 & (v) & \\
\hline Operation Theater & 1.1 & 23-Nov-2011 & (v) & $\begin{array}{l}\text { - } 0 \text { I\%20Status\%20Transactionwise.doc } \\
\text { - Amendment } \\
\text { \%20\%200T\%20module.docr }\end{array}$ \\
\hline Diet Kitchen & 4.1 & 23-Nov-2D11 & (v) & $\begin{array}{l}\text { - } \underline{D K} \text { transartinnFinal FR1 } \\
\text { - } \underline{\text { DK MST.ER1 }}\end{array}$ \\
\hline Line And Laundry & 1 & 04-Nov-2011 & (v) & \\
\hline Hem (Bmed) & 3 & 14 -Jun-2011 & (v) & \\
\hline
\end{tabular}

Figure 6: Trace change request design document

\subsection{CR Upload}

Before the development of this utility, all CR Design document are created on MS Word Editor. This process is used to upload such manual design created so that tracking of design changes are also online. 


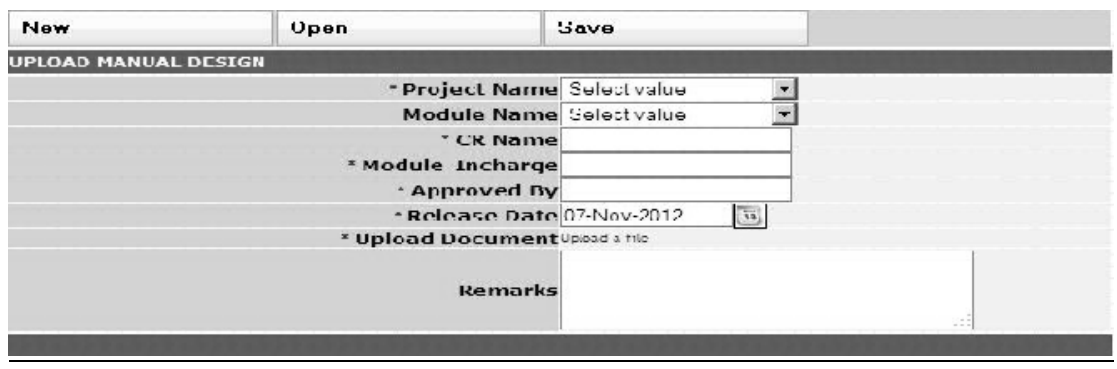

Figure 7: CR Upload

\section{TECHNiCAL IMPLEMENTATION ASPECTS OF CR DESIGNER}

CR Designer is developed using J2EE Technology with oracle as the Database. In order to provide security and authenticity to the data the architecture is divided into three layers. Each layer has specified representation.[7] These layers are described as:

\subsection{Presentation Layer}

This layer is used by the user for interaction with the system. It is used to manage the way the data to be viewed in jsp. Values of form bean request and session are managed through presentation layer. The function of this layer is to take the inputs form the client browser and to send the output to the client browser. This layer interacts with the business layer for logics.

\subsection{Business Layer}

This layer is used for business logics . it takes data form presentation layer as well as Data Access layer. The methods in business layer calls data access layer functions and after getting result it process the results according to the requirement of presentation layer and sends the final result to the presentation layer

\subsection{Data Layer}

Data access layer contains methods which are used to insert and update data to the database with the help of stored procedures and queries in the form of property files 


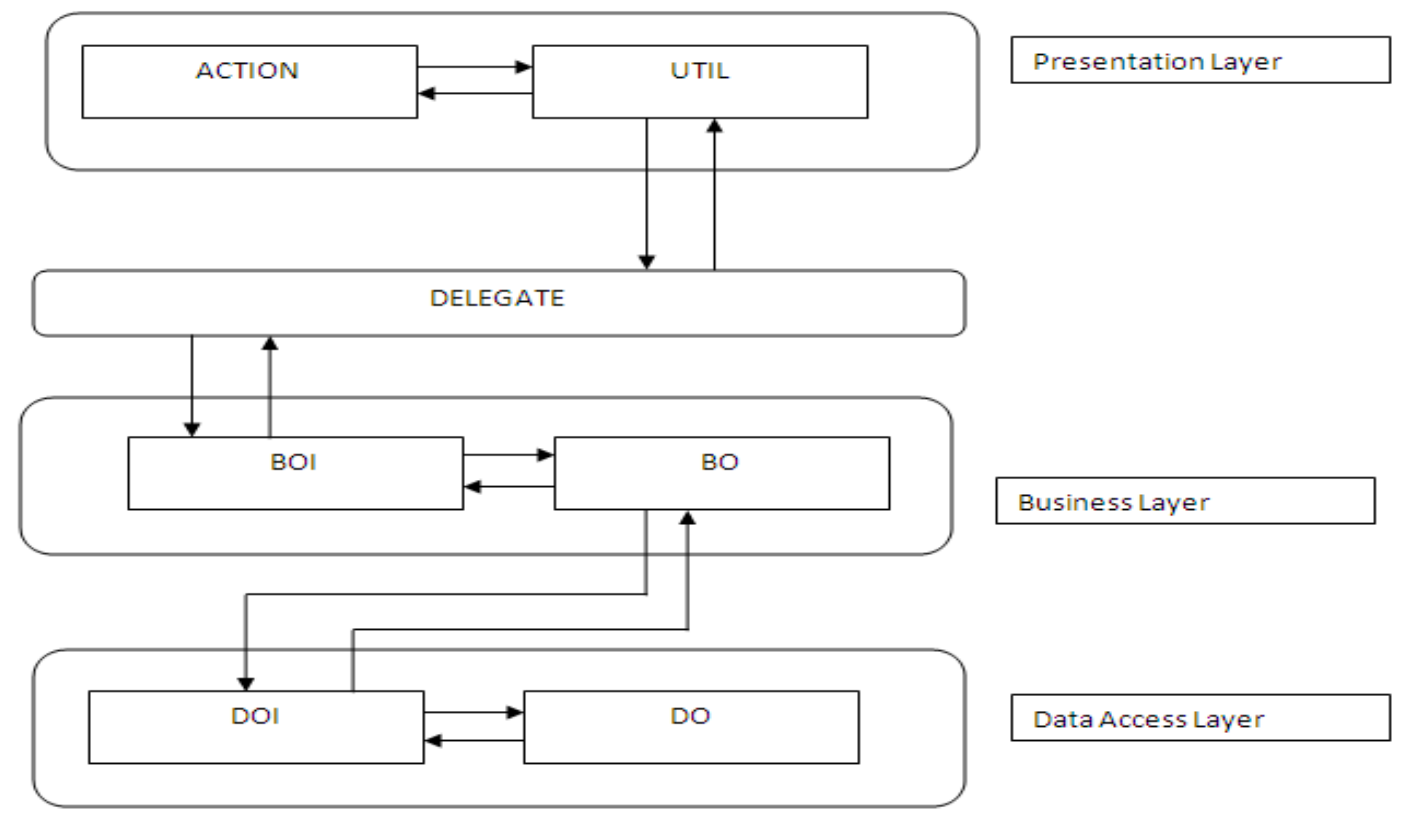

Figure 8: Application Internal Architecture

\section{CONCLUSION}

The software Engineering design documentations is prepared for development of utility. But least effort is made after implementation of utility. Most of the time design documentation is bypass and directly development/customization is done. The CR Design documentation will help in tracking and keeping in account design changes done in the S/w system after implementation also.

CR Designer tool helps in fast Creator of CRD document. This tool allows user friendly Creator and modification of CRD documents. It also saves the time and effort of the designer for Creator of CRD documents. A CRD document undergoes multiple iterations during its Creator. The tool therefore also cares of the versioning issues. The tool maintains a repository of all the released CRD Documents and help in tracking the same. This tool also maintains all the CR documents which are created in MS-word.

\section{ACKNOWLEDGMENT}

We express our deep thanks to Mr. B.K. Murthy, Executive Director; CDAC Noida (India), with the help of his vast experience, efforts, valuable suggestion, support and motivation helped us to great extent for the design and development of this utility.

\section{REFERENCES}

[1] http://www.cdacnoida.in/ascnt2011/quality\%5C3.\%20Low\%20Level\%20Design\%20Document\%20C reator\%20Tool.pdf

[2] http://en.wikipedia.org/wiki/Systems_Development_Life_Cycle

[3] Research Paper: - CiteSeer

[4] http://www.alistapart.com/articles/gettingrealaboutagiledesign/

[5] http://en.wikipedia.org/wiki/Interaction_design 
[6] http://quality-assurance-software-testing.blogspot.in/2006/12/what-is-traceability-matrix.html

[7] http://www.cdacnoida.in/ASCNT-2012/ASCNT-2012/EGOV/4.pdf

[8] http://seij.dce.edu/vol_2_issue_2/paper2.pdf

[9] http://www.cs.washington.edu/education/courses/csep504/10wi/Cheng09.pdf

[10] http://www.upedu.org/process/gcncpt/co_revno.htm

[11] http://en.wikipedia.org/wiki/Systems_development_life-cycle

[12] http://en.wikipedia.org/wiki/Change_request

\section{Authors}

\section{Siddharth Dixit}

$\mathrm{He}$ is working as Senior Engineer (IT) at CDAC Noida. He has 6 years of IT Experience, in various Health Informatics projects. He had completed Master in.Technology from Amity University, Noida India. He has also done his PG Diploma in Advanced Software Design and Development from CDAC, Noida. He has expertise in Analysis and Designing of Health Informatics Application. Research Oriented Software Design and Development is his key interest area.

Email: siddharthdixit@cdac.in

\section{Ranu Ratnawat}

She is working as Junior Research Fellow at CDAC Noida. She has 1 years of IT Experience, in various Health Informatics projects. She has completed her Masters in Computer Application from Banasthali University, India.Mobile based application development is her key interest area. Email: ranuratnawat@cdac.in

\section{Umesh Sharma}

He has completed his Masters of Computer Application from GGSIP University, Delhi. Currently he is working as Technical Officer in CDAC. His achievements includes:-

Successful implementation of Hospital Information Management Information (HIMS) Application in Indira Gandhi Medical College (IGMC), Shimla, India.

Design, Development and implementation of Laboratory Information System module at Post Graduate Institute Medical Education \& Research (PGIMER) Chandigarh, GNCTD Delhi

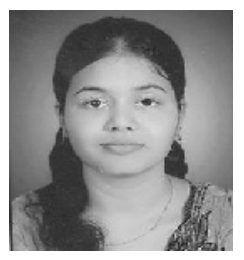

Design and Development of Patient Medical Record Department Module for MGIMS Nagpur Design and Development of various modules of HIS for PGIMER Chandigarh, GNCTD Delhi \& RAOL Rajasthan. Email: umeshsharma@cdac.in

\section{Praveen Kr Srivastava}

He has done his Masters in Computer Application from Madan Mohan Malviya Engineering College Gorakhpur. Currently he is working as Joint Director and Group Coordinator for Design Group in CDAC Noida.

His achievements include:

He was the Topper of M.Sc (Maths) from Allahabad University He is having around

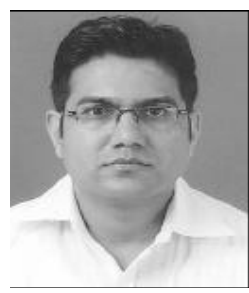

15 Years of Experience in the Development and implementation of Health Care Solutions at Different Hospitals. He has deep interest in standardizing the Electronic Medical Record in Hospitals. Email: pksrivastava@cdac.in 